\title{
Hipotiroidismo en tratamiento en el Área de Salud de Guadalajara (España): características y prevalencia estimadas a partir del consumo de hormona tiroidea
}

\author{
$\mathrm{M}^{\mathrm{a}}$ Carolina Marí Silva ${ }^{\mathrm{a}}$, Petronela Toderescu ${ }^{\mathrm{a}}$, José $\mathrm{M}^{\mathrm{a}}$ Alonso Gordo $\mathrm{M}^{\mathrm{a}}$ Isabel Pérez Rodríguez, \\ $M^{\mathrm{a}}$ Carmen Sánchez González ${ }^{\mathrm{a}}$, Alina Dumbraveanu ${ }^{\mathrm{a}}$
}

${ }^{a}$ Centro de Salud GuadalajaraSur (Guadalajara).

b Sección de Farmacia. Gerencia de Atención Primaria del SESCAM. (Guadalajara)

Correspondencia: José $\mathrm{M}^{\mathrm{a}}$ Alonso Gordo. Centro de Salud Guadalajara-Sur. C/Ferial, 31, 19002 - Guadalajara. Tfno: 949226508 . FAX 949219667. Correo electrónico: jmalonso@sescam.org.

Recibido el 20 de febrero de 2012.

Aceptado para su publicación el 21 de marzo de 2012.

\section{RESUMEN}

Objetivo. Nos proponemos estimar la prevalencia del hipotiroidismo tratado en nuestra Área de Salud a partir de la prescripción de hormona tiroidea. Describimos asimismo sus características y diferencias en base a diversos factores sociodemográficos.

Diseño. Estudio descriptivo de corte transversal.

Emplazamiento. Área Sanitaria de Guadalajara (España).

Participantes. Sujetos en tratamiento con levotiroxina por hipotiroidismo incluidos en tarjeta sanitaria durante el año 2009.

Mediciones Principales. Se evaluaron la distribución por sexo, edad y lugar de residencia, previo ajuste de tasas. Otras variables analizadas fueron el tipo de hipotiroidismo, última dosis prescrita y niveles de TSH, comparando en función de variables sociodemográficas.

Resultados. Un 3,3\% de la población (IC 95\%: 3,3-3,4) recibió tratamiento con levotiroxina ( $5,8 \%$ mujeres y $1,0 \%$ varones), observando la tasa más elevada en mujeres mayores de 65 años (11,9\%). La tasa ajustada fue 38,8/1000 (IC 95\%: 37,7-40,0) para el medio urbano y 34,6/1000 (IC 95\%: 33,4-35,8) para el rural. Edad media de 56,6 años (DE: 18,2). Se asume origen autoinmune en el $76 \%$ de los casos con causa reconocible, utilizando una dosis media de $76,84 \mathrm{mcg} / \mathrm{día}$. Un $76,2 \%$ de los pacientes presentaron un rango correcto de TSH.

Conclusiones. El hipotiroidismo subclínico es un proceso frecuente entre los usuarios de un Centro de Salud y mayoritariamente tiene origen autoinmune. El diagnóstico es más frecuente en el sexo femenino y en población mayor. Existe habitualmente un buen control de las cifras de TSH. Las diferencias geográficas son poco relevantes.

Palabras clave. Hipotiroidismo, Hipotiroidismo subclínico, Prevalencia, Atención Primaria, Levotiroxina.

\section{ABSTRACT}

Hypothyroidism under treatment in the Guadalajara (Spain) health care area: characteristics and prevalence based on the intake of thyroid hormone

Objective. It is our aim to estimate the prevalence of hypothyroidism treated in our health care area from the prescription of thyroid hormone. Likewise, we describe its characteristics and differences on the basis of various socio-demographic factors.

Design. Cross sectional descriptive-type study.

Location. Guadalajara (Spain) health care area.

Participants. Subjects under treatment with levothyroxine for hypothyroidism included on health card throughout the year 2009.

Main Measures. Distribution by gender, age and place of residence, after rate adjustment. Other analyzed variables were the type of hypothyroidism, last prescribed dosage and TSH levels, compared according to socio-demographic variables.

Results. 3,3\% of the population (IC 95\%: 3,3-3,4) were treated with levothyroxine $(5,8 \%$ female y $1,0 \%$ male), with the highest rate being seen in women over 65 years old $(11,9 \%)$ The adjusted rate was 38,8/1000 (IC 95\%: 37,7-40,0) for urban areas and 34,6/1000 (IC 95\%: $33,4-35,8$ ) for rural areas. Average age 56,6 years (DE: 18,2 ). An autoimmune origin is assumed in $76 \%$ of cases with a recognizable cause, using an average dose of $76,84 \mathrm{mcg} /$ day. $76,2 \%$ of the patients showed a correct level TSH.

Conclusions. Subclinical hypothyroidism is a frequent complaint among Health Centre users and has a chiefly autoimmune origin. Diagnosis is more frequent in females and in older members of the population. TSH figures are usually closely checked. Geographical differences of little significance.

Key words. Hypothyroidism, subclinical hypothyroidism, prevalence, primary health care, levothyroxine. 


\section{INTRODUCCIÓN}

Las alteraciones en el funcionamiento de la glándula tiroides, por hiper o hipotiroidismo, son un hallazgo bastante frecuente en las consultas de Atención Primaria, pudiendo encontrarse cifras de prevalencia entre $5,9 \%$ y $9,1 \%$ de la población general adulta ${ }^{1,2}$, y considerando también márgenes bastante amplios ${ }^{3}$ esta disfunción funcional tiroidea puede oscilar en ancianos entre el 8,5 y el $13 \%{ }^{4-6}$. La frecuencia de hipertiroidismo es muy inferior a la de hipotiroidismo $0^{1-3,7-9}$.

El hipotiroidismo se puede presentar en su forma mixedematosa, sintomática y poco habitual, pero también puede presentarse de forma silente $u$ oligosintomática, (denominado hipotiroidismo subclínico), con cifras de TSH elevadas y normales de T3 y T4. Ésta es la forma más frecuente de disfunción de esta glándula, afectando a un $1-10 \%$ de la población ${ }^{1,3,10-16}$, aunque en personas mayores puede ser superior al $10 \% \cdot{ }^{17}$ Es conocido que el sexo femenino está afectado con mayor frecuencia, de modo que en hombres jóvenes se publican cifras del $0,1 \%{ }^{18}$, pero pueden ser superiores al $20 \%$ en mujeres de mayor edad ${ }^{14}$.

El sustrato fisiopatológico radica en una disminución en la producción de hormonas tiroideas, ya sea por una alteración de la glándula tiroidea (hipotiroidismo primario) o por una disminución de su estimulación a nivel hipotalámico o hipofisario (hipotiroidismo secundario o terciario). ${ }^{19}$ La etiología más frecuente de hipotiroidismo tiene carácter autoinmune ${ }^{3,14}$, si bien también son relativamente frecuentes los cuadros secundarios a ablación quirúrgica 0 radioterápica, los secundarios a fármacos ${ }^{3,8,20}$ y los que acompañan a otras enfermedades agudas 0 crónicas $^{14}$.

Los datos de prevalencia del hipotiroidismo subclínico presentan diferencias considerables dependiendo de la población estudiada, punto de corte de TSH y del diseño metodológico, aumentando la variabilidad si se añaden otros factores como edad, sexo o ámbito geográfico.

La prevalencia de hipotiroidismo puede estudiarse utilizando los métodos directos mediante estudios poblacionales con determinaciones hormonales, que son exactos, pero también costosos. Los métodos indirectos, como el de este estudio, únicamente nos ofrecen estimaciones, sin embargo su viabilidad es superior.
En nuestro país se han publicado algunos estudios sobre prevalencia de hipotiroidismo, con resultados también variables dependiendo del ámbito, población y metodología. Mediante determinaciones analíticas en población adulta se han obtenido cifras de hipotiroidismo clínico o subclínico entre 2,4 y 3,5\% $\%^{1,2}$. En cambio, utilizando la Dosis Diaria Definida de las hormonas tiroideas, se han estimado valores entre 4 y 9,3 por 1.000 habitantes ${ }^{21-23}$, siendo Guadalajara la provincia que presenta las cifras más altas en uno de los trabajos consultados $(5,02$ y 7,01 casos por 1.000 habitantes en 1996 y 1999 respectivamente) $)^{21}$.

Dado que actualmente el tratamiento del hipotiroidismo se realiza con un único producto, levotiroxina, es posible estimar la prevalencia del hipotiroidismo tratado (clínico o subclínico), conociendo el número de personas a los que se prescribe. Mediante este método se han publicado cifras de prevalencia que oscilan entre el 1 y el $2 \%{ }^{21-25}$.

Dada la disparidad de los datos publicados, la sospecha de una prevalencia de hipotiroidismo más elevada que la publicada y la escasez de estudios en población general en los últimos años en nuestro medio, nos proponemos estimar la prevalencia de hipotiroidismo, en base al tratamiento con hormonas tiroideas en pacientes con tarjeta sanitaria del Área de Salud (AS) de Guadalajara. Asimismo, describir las características de estos casos y estimar si los factores, como edad, sexo y lugar de residencia, influyen en el número de casos de hipotiroidismo y en sus características.

\section{MATERIAL Y MÉTODOS}

El Área Sanitaria (AS) de Guadalajara tenía 230.695 personas con tarjeta sanitaria a finales del año 2009, habiéndose excluido del estudio las personas sin tarjeta. Dicha población se distribuía en 6 centros de salud urbanos y 23 centros de salud rurales (de los que 11 pertenecen a las denominadas Serranías), dependientes de la Gerencia de Atención Primaria del SESCAM (Servicio de Salud de Castilla la Mancha). El Servicio de Farmacia de dicha Gerencia posee datos informatizados de todas las prescripciones realizadas por los profesionales (hospitalarios y de Atención Primaria) del AS, una vez retiradas de las oficinas de farmacia. A partir de dicha información, 
y de la historia clínica informatizada, se identifican los pacientes en tratamiento con Levotiroxina y se obtienen datos sociodemográficos y clínicos y de sus centros de salud de referencia.

Con dichos datos hemos realizado un primer estudio observacional, descriptivo, de corte transversal, de las dispensaciones de Levotiroxina, en cualquiera de sus formas comerciales, efectuadas por todas las oficinas de farmacia de la provincia de Guadalajara, con receta de la Seguridad Social durante el año 2009. El cálculo de la prevalencia se realiza diferenciando y estableciendo valores por lugar de residencia, centro de salud, edad y sexo. Se calculan las tasas brutas y posteriormente se realiza un ajuste de tasas mediante método indirecto, con el programa de análisis epidemiológico de datos tabulados (EPIDAT) versión 3.1, utilizando como población estándar la población española en 2009, según el padrón del Instituto Nacional de Estadística; se compara medio urbano o rural (por encima o debajo de 10.000 habitantes), y distinguiendo en éste también las comarcas naturales (Serranías o Alcarria-Campiña). Se calculan intervalos de confianza al 95\% para los grandes grupos demográficos y para los centros de salud más importantes. De los centros con escasa población (menos de 2500 habitantes) y escasa casuística, en su mayor parte de la comarca de la sierra, no presentamos datos, dada, además, su alta variabilidad y elevada distorsión en el ajuste de tasas.

En la segunda parte del estudio se realiza un muestreo aleatorio sistemático de los 7.717 pacientes en tratamiento con Levotiroxina. Considerando una prevalencia de hipotiroidismo de un $2 \%{ }^{21-25}$, una precisión de $\pm 1 \%$ y una confianza del $95 \%$ el tamaño calculado fue de 686 sujetos, obteniéndose un tamaño muestral definitivo 717 pacientes. De ellos 114 fueron hombres $(15,9 \%)$ y 603 mujeres $(84,1 \%)$, evaluándose en ellos las características sociodemográficas, confirmación del diagnóstico, tipo de hipotiroidismo, último valor analítico de TSH, valor de anticuerpos antitiroideos, dosis actual de Levotiroxina recetada y fármacos asociados. Los valores de estas variables se obtuvieron a través de la búsqueda en la historia clínica informatizada por el denominado programa "Turriano" del SESCAM, implantado en todos los Centros de Salud; los datos analíticos se obtuvieron del propio sistema Turriano y del laboratorio de análisis clínicos del Hospital Universitario de Guadalajara.

El análisis de los datos se ejecuta mediante el programa estadístico SPSS versión 17, y EPIDAT versión 3.1. Se analizan las frecuencias, se calculan las medias y proporciones de las diferentes variables con sus intervalos de confianza al 95\% y Desviación Estándar (DE). Se analizó la asociación entre variables utilizando test paramétricos como la $T$ de Student y Chi-Cuadrado dependiendo del tipo de variable cuantitativa o cualitativa, respectivamente.

\section{RESULTADOS}

En el periodo analizado (año 2009) estaban incluidos en el AS de Guadalajara 7.717 pacientes entre 1 y 99 años de edad en tratamiento con Levotiroxina, de los que $1.200(15,5 \%)$ eran hombres. La edad media de los pacientes fue de 56,64 años (DE $18,2)$, con 58,1 años para hombres y 56,4 para mujeres $(p<0,05)$.

La prevalencia del hipotiroidismo tratado fue de 3,34\% (IC 95\%: 3,27-3,41). Se observa mayor prevalencia en mujeres que en hombres $(5,8 \%$ vs $1,0 \%$, respectivamente; $p<0,01)$. Las tasas brutas

\begin{tabular}{l|cccc}
\hline & HOMBRES & MUJERES & TOTAL/1000 & PROPORCIÓN M/H \\
\hline 0-14 años & 1,02 & 2,48 & 1,73 & 1,43 \\
15 a 64 años & 8,37 & 55,26 & 32,01 & 6,6 \\
> 65 años & 32,45 & 118,75 & 76,25 & 3,65 \\
TOTAL & 10,30 & 57,98 & 33,45 & 5,63 \\
\hline
\end{tabular}

Tabla 1. Tasas brutas/1000 h. por grupos de edad y sexo. M/H: proporción mujeres/varones 


\begin{tabular}{l|lll}
\hline & HOMBRE (IC 95\%) & MUJER (IC 95\%) & TOTAL (IC 95\%) \\
\hline SIERRA & $9,02(7,29-10,75)$ & $66,09(60,56-71,62)$ & $33,85(31,27-36,42)$ \\
NO SIERRA & $11,25(10,57-11,93)$ & $64,46(62,78-66,14)$ & $37,31(36,41-38,20)$ \\
CAMPI/ALCARRIA & $9,98(8,97-10,98)$ & $62.82(60,11-65,54)$ & $34,99(33,6-36,37)$ \\
RURAL & $9,71(8,85-10,57)$ & $63,15(60,75-65,55)$ & $34,62(33,42-35,83)$ \\
URBANO & $12,05(11,13-12,98)$ & $65,44(63,28-67,60)$ & $38,85(37,67-40,02)$ \\
\hline
\end{tabular}

Tabla 2. Tasas ajustadas/1000 habitantes por comarcas.

\begin{tabular}{l|cc}
\hline & $\begin{array}{c}\text { HOMBRE } \\
\text { TASAS AJUSTADAS }\end{array}$ & $\begin{array}{c}\text { MUJER } \\
\text { TASAS AJUSTADAS }\end{array}$ \\
\hline AZUQUECA & $12,9(11,6-14,8)$ & $68,2(63,9-72,4)$ \\
BRIHUEGA & $18,1(12,8-23,4)$ & $73,5(61,6-85,5)$ \\
CABANILLAS & $8,5(5,5-11,6)$ & $64,9(56,5-73,5)$ \\
CIFUENTES & $13,0(8,0-18,1)$ & $80,7(67,5-94,5)$ \\
EL CASAR & $9,6(7,0-12,3)$ & $52,5(46,1-58,9)$ \\
GUADALAJARA & $11,9(10,8-13,0)$ & $64,3(61,7-66,8)$ \\
HORCHE & $5,5(2,2-8,9)$ & $58,0(46,6-69,3)$ \\
JADRAQUE & $8,0(3,4-12,6)$ & $77,1(60,4-93,8)$ \\
LA CAMPIÑA & $10,8(6,7-14,9)$ & $66,5(55,8-77.3)$ \\
MOLINA & $9,4(5,8-13,0)$ & $54,4(52,0-72,9)$ \\
MONDÉJAR & $7,9(4,9-10,8)$ & $67,8(56,4-63,0)$ \\
PASTRANA & $8,2(4,9-11,6)$ & $73,2(63,6-89,2)$ \\
PERIFÉRICO & $10,8(7,2-14,3)$ & $59,1(48,5-69,7)$ \\
SACEDÓN & $10,6(6,6-14,6)$ & $68,5(58,5-78,5)$ \\
SIGÜENZA & $10,2(6,9-13,5)$ & $62,4(54,0-70,8)$ \\
YUNQUERA & $9,7(6,7-12,8)$ & \\
\hline
\end{tabular}

Tabla 3. Tasas ajustadas/ 1.000 h. por Centros de Salud (IC 95\%)

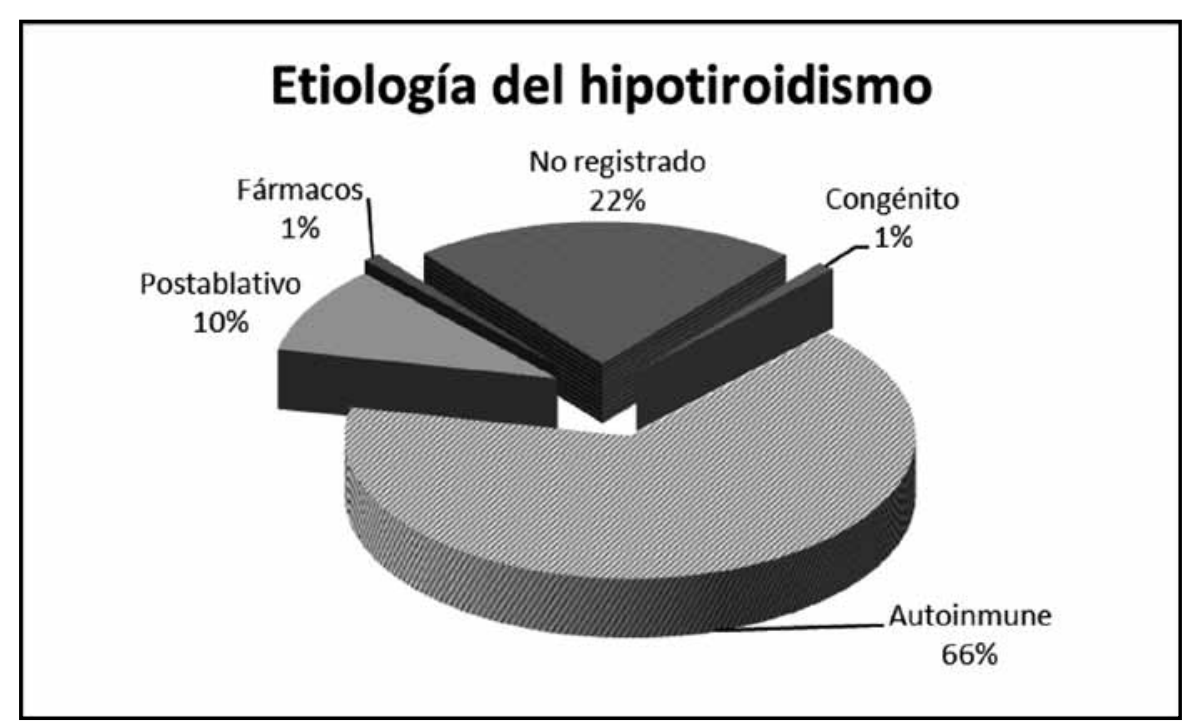

Fig. 1. Distribución de los tipos etiológicos de hipotiroidismo. 


\begin{tabular}{|c|c|c|c|c|c|c|}
\hline AUTOR & AÑo & MÉTODO & POBLACIÓN & HOMBRES & MUJERES & TOTAL \\
\hline AYALA $^{26}$ & 2001 & TSH & $>65 a$ & 4 & 6,3 & 5,4 \\
\hline $\mathrm{BJORO}^{7}$ & 2000 & ENCUESTA & $>20 \mathrm{a}$ & 0,9 & 4,8 & \\
\hline $\mathrm{BJORO}^{7}$ & 2000 & TSH & $>20 \mathrm{a}$ & 4,07 & 6 & \\
\hline BRACERAS ${ }^{23}$ & 2007 & $\mathrm{DHD}$ & GENERAL & & & 0,93 \\
\hline DIAZ MAD 22 & 2001 & DDD & GENERAL & & & 0,49 \\
\hline $\mathrm{DIEZ}^{27}$ & 2003 & TSH & $>60 \mathrm{a}$ & 0 & 4,72 & 2,04 \\
\hline EXCUER $^{2}$ & 1999 & $\mathrm{TSH}$ & $>6 a$ & & & 3,48 \\
\hline HUNTER ${ }^{18}$ & 2000 & PRESCRIPCION & $<22 \mathrm{a}$ & 0,06 & 0,15 & 0,14 \\
\hline MAESTRO 28 & 2005 & TSH & $>75 a$ & 10,4 & 16,4 & 13,8 \\
\hline MORANT $^{21}$ & 2001 & DHD & GENERAL & & & 4,33 \\
\hline RIVOLTA $^{13}$ & 1999 & TSH & $>17 \mathrm{a}$ & 3,4 & 6,1 & 4,7 \\
\hline SAWIN ${ }^{17}$ & 1985 & TSH & $>60 \mathrm{a}$ & 5,7 & 13,6 & 10,3 \\
\hline SEMPERE ${ }^{25}$ & 2005 & PRESCRIPCION & $>14 \mathrm{a}$ & 0,4 & 2,3 & 1,4 \\
\hline SENDER ${ }^{4}$ & 2004 & TSH & $>60 \mathrm{a}$ & 4,7 & 15,8 & 13 \\
\hline SENDER ${ }^{1}$ & 2002 & $\mathrm{TSH}$ & $>14 \mathrm{a}$ & 1,4 & 6,3 & 5.4 \\
\hline SERNA ${ }^{24}$ & 2003 & DHD & GENERAL & 0,24 & 1,24 & 0,84 \\
\hline NOSOTROS & 2009 & PRESCRIPCIÓN & GENERAL & 1,03 & 5.8 & 3,34 \\
\hline
\end{tabular}

Tabla 4. Prevalencia de hipotiroidismo (\%) según diversos autores y métodos.

por 1.000 habitantes por sexo y grupo de edad se observan en la tabla 1.

Se observó una prevalencia bruta de hipotiroidismo superior, aunque sin alcanzar la significación estadística, en la zona rural $(3,40 \%)$ que en la urbana $(3,30 \%)$, mientras que fue significativamente más elevada en la zona de sierra que en la zona de no sierra $(4,15$ vs $3,28 \% ; p<0,01)$, manteniéndose las diferencias por sexo. No obstante, las cifras se modifican una vez ajustadas las tasas en función del lugar de residencia, como se puede observar en la tabla 2. La tasa ajustada fue de 38,8/1000 (IC 95\%: 37,7-40,0) para el medio urbano y de 34,6/1.000 (IC 95\%: 33,4-35,8) para el medio rural $(p<0,01)$, no observándose diferencias en función de pertenecer o no a la zona de sierra.

En la tabla 3 se muestran las tasas ajustadas por sexo para los Centros de Salud de mayor población o con mayores tasas de prevalencia. Podemos observar en todos los casos diferencias por sexo a favor de las mujeres en una proporción similar y significativa $(p<0,01)$.

La distribución por tipo de hipotiroidismo se puede apreciar en la figura $n^{\circ} 1$, destacando el $66,4 \%$ de origen autoinmune $(76 \%$ de los que consta etiología). Se detectaron 11 casos $(1,5 \%)$ en los que no se pudo constatar el diagnóstico de hipotiroidismo, a pesar de haber recibido alguna prescripción de Levotiroxina, y en un $22,2 \%$ no se encontraron datos suficientes para poder determinar la etiología del hipotiroidismo.

La dosis media de tratamiento fue de $76,84 \mathrm{mcg} /$ día (DE:18,00), con una dosis mínima de 25 y una máxima de 200. La dosis más frecuentemente utilizada fue de $50 \mathrm{mcg} /$ día $(28,9 \%)$, seguida por la de $100 \mathrm{mcg} /$ día (20,8\%), y la de 75 mcg/día (17,9\%). Las dosis intermedias utilizadas fueron de $62 \mathrm{mcg}$, $88 \mathrm{mcg}$ y $112 \mathrm{mcg}$. No se observaron diferencias 
estadísticamente significativas en la dosis media de hombres y mujeres.

El $1 \%$ de los pacientes tomaba Amiodarona, fármaco potencialmente inductor de disfunción tiroidea, y no encontramos pacientes que estuvieran tomando otra medicación habitualmente relacionada con disfunción tiroidea (litio...).

En función de la última cifra de $\mathrm{TSH}$, se puede considerar que más del $75 \%$ de los pacientes se encontraban en rango terapéutico, siendo el grado de control el siguiente: un $9,7 \%$ se consideraron hipertratados $(\mathrm{TSH}<0,37 \mathrm{mU} / \mathrm{l})$, un $76,2 \%$ bien controlados (TSH 0,37-4,7mU/l) y el $14,1 \%$ se consideraron como posiblemente infradosificados (TSH $>4,7 \mathrm{mU} / \mathrm{l})$. No se observaron diferencias respecto al control entre los pacientes del medio rural y los del medio urbano. Mientras que sí se mostraron diferencias estadísticamente significativas $(p<0,005)$ en cuanto al rango de control según valores de TSH y sexo, observándose valores fuera de rango más frecuentemente en los hombres (OR: 2,02; IC 95\%: 1,30-3,13).

\section{DISCUSIÓN}

Diversos estudios han abordado la estimación de la prevalencia de hipotiroidismo en base al consumo de hormonas tiroideas en nuestro país ${ }^{22-24}$, así mostramos en la tabla $n^{\circ} 4$ las prevalencias, en tanto por ciento, publicadas por diversos autores, el año de estudio, el grupo de edad y la metodología utilizada, con una amplia variabilidad en función de estos factores.

La prevalencia de hipotiroidismo tratado en nuestro caso es del 3,34\% en población general, siendo superior a la de otros estudios publicados ${ }^{1,21-24}$. La distribución por sexo es similar a la publicada por otros autores ${ }^{2,7,21}$, siendo superior en las mujeres, como se observa en la mayor parte de los estudios. Aunque sospechamos una prevalencia de hipotiroidismo en nuestra AS superior a otras publicadas, hecho ya referido en un estudio previo ${ }^{21}$, no podemos conocer la prevalencia real de hipotiroidismo, pues es una estimación basada en prescripción en el sistema sanitario público y sólo estudiamos a población con tarjeta sanitaria. Además, pueden existir casos sin diagnosticar ni tratar, teniendo en cuenta que en la mayor parte de los pacientes se trata de hipotiroidismo subclínico, y que, incluso, la mayor frecuencia de determinación de hormona tiroidea en las mujeres, podría influir en la diferencia por sexos ${ }^{1}$.

En un intento de depuración de la información, y para el estudio de las características del hipotiroidismo, se confrontaron los datos de la muestra de 717 pacientes, encontrándose sólo 11 casos $(1,5 \%)$ en los que el deficiente registro de los datos podría hacer dudar del diagnóstico de hipotiroidismo, aunque a dichos pacientes se les había prescrito alguna dosis de Levotiroxina. Es posible que el fármaco fuera destinado, puntualmente, a otra persona del núcleo familiar. También hemos encontrado 159 casos (22\%) en los que, aun no habiendo duda sobre el diagnóstico, no queda clara la etiología en función de los antecedentes o de la inexistencia de valores registrados de anticuerpos antitiroideos.

Otras limitaciones del estudio vendrían dadas por el hecho de que algún paciente con hipotiroidismo no reciba recetas de la Seguridad Social. En nuestro caso el 1,4\% de los pacientes estudiados pertenece a otros ámbitos de prescripción (ISFAS, MUFACE), lo que debe introducir un factor de corrección posiblemente al alza en la prevalencia de hipotiroidismo, como ya han apuntado otros autores $^{22,24}$. Finalmente, es posible que algún paciente con tarjeta sanitaria no adquiera la medicación mediante receta de la SS o la retire ocasionalmente fuera del AS, pero la posibilidad de que no la haya retirado en ningún momento del año 2009 en las oficinas de farmacia del Área es escasa, por lo que pensamos que nuestra estimación es bastante próxima a la realidad.

La tasa de hipotiroidismo es baja en pacientes jóvenes $\left(1,35 / 1000\right.$ según Hunter ${ }^{18}$ y 1,73 en nuestro estudio), y aumenta significativamente con la edad. Todos los estudios revisados, al igual que el nuestro, ofrecen mayores prevalencias en personas mayores, presentando cifras que en su mayoría oscilan entre el 7 y el $12 \%{ }^{4,11,29}$, habiéndose publicado cifras del $13,8 \%$ en nuestra Area de Salud $^{28}$. Este incremento etario parece lógico, dado que estamos hablando de un padecimiento crónico, con un tratamiento habitualmente de por vida y, por tanto, los casos son acumulativos.

Es difícil explicar las diferencias encontradas entre los diversos centros de salud y comarcas, si bien el ajuste de tasas corrige o minimiza las diferencias debidas a la pirámide de población, esto no siempre repercute de igual manera en todas las poblaciones, sobre todo cuando la población y la casuística por décadas es muy baja. Algunos centros de salud, como Brihuega o Cifuentes, tienen las tasas ajustadas más elevadas del Área, siendo Horche y Mondéjar los que las presentan más bajas, sin aparentes factores objetivos que justifique las diferencias tan elevadas. Por otro lado, parece que la zona de las Serranías tiene tasas 
más altas que las comarcas que no pertenecen a esta zona y a ello podría contribuir la habitual asociación epidemiológica con la carencia de yodo en la alimentación y mayor frecuencia de bocio. Sin embargo, al ajustar tasas, es el medio urbano el que tiene tasas más altas de modo significativo en el caso de la tasa total y de los hombres, mientras que en las mujeres no existe diferencia. Aunque se han publicado datos de carencia importante de yodo en la población ${ }^{30}$, posiblemente en la época actual el consumo de yodo esté más equiparado entre residentes en las diversas comarcas y ya no tenga un peso específico tan elevado en la génesis del hipotiroidismo.

Hemos intentado aproximarnos también a la posibilidad de que otros factores geográficos, incluido la proximidad a la centrales nucleares, tuviera alguna relación, sin que hayamos podido encontrar datos de sospecha en este sentido (datos no presentados). Por un lado la movilidad poblacional actual por razones familiares, laborales o académicas es elevada y sería arriesgado intentar establecer una correlación entre la residencia actual y el factor de riesgo geográfico, por otro lado una Zona de Salud puede tener núcleos poblacionales a más de $50 \mathrm{~km}$ entre sí por lo que al posible factor de exposición habría que aplicarle un factor de corrección, haciendo que cualquier conclusión en este sentido fuera muy arriesgada y bastante inexacta.

Otros factores que podrían influir en las diferencias (respecto a otros estudios o entre comarcas del AS) estarían en relación con la accesibilidad a los servicios sanitarios, capacidad de captación de casos subclínicos o incluso una mayor agresividad diagnóstica en base a riesgos de otras patologías (obesidad, dislipemias...). Estas diferencias pueden sospecharse incluso en diferentes cupos médicos de un mismo centro de Salud ${ }^{25}$, aunque detectar la causa real de la diferencia requeriría otro tipo de estudios epidemiológicos poblacionales.

El hipotiroidismo autoinmune representa en nuestro caso el $66,4 \%$ de todos los casos $(76 \%$ de las que tiene causa reconocible), cifra que podría ser superior, dado que la mayor parte de los 159 casos sin datos suficientes podrían corresponder a este grupo y también algunos de los debidos a cirugía o radioterapia por tratamiento de hipertiroidismo autoinmune. La etiología autoinmune del hipotiroidismo, publicada por otros autores, cifra también el porcentaje entre el 47 y el $53 \% \%^{6,29,31}$, alcanzando el $58,6 \%$ en el caso del hipotiroidismo subclínico ${ }^{31}$, siendo también conocido que las enfermedades autoinmunes, y también las tiroideas, son más frecuentes en la población femenina.

La dosis media diaria utilizada en nuestra población $(76,8 \mathrm{mcg})$ está muy próxima al rango recomendado de 50 a $75 \mathrm{mcg} / \mathrm{día}^{14}$, aunque la DDD es en nuestro medio de $150 \mathrm{mcg}^{24}$. En cuanto al grado de control (76,2\% bien controlados), es algo superior a otros resultados publicados $(67,5 \%$ en un estudio realizado en Madrid en $2002^{31}$ y $60 \%$ en el estudio de Colorado $^{16}$ ). No se observaron diferencias significativas entre los tipos de hipotiroidismo y el grado de control según la TSH en el medio rural y urbano. En cambio, y a diferencia del estudio anterior, sí se observa que los varones de nuestro estudio están con valores de TSH fuera del rango óptimo más frecuentemente que las mujeres, sugiriendo que ser hombre aumenta el doble la posibilidad de tener un valor de TSH fuera del rango eutiroideo. Es posible que la mayor frecuentación de la mujer a los servicios asistenciales explique parte de las diferencias en relación con la prevalencia de hipotiroidismo diagnosticado y con el grado de control conseguido.

Cuestión diferente, y que no hemos estudiado, sería valorar la adecuación del tratamiento en función de los datos y de los riesgos de tratar o no tratar. La aplicación individualizada de algoritmos de decisión, la valoración global del riesgo de cada paciente y el seguimiento clínico, nos ayudarán a adoptar las actuaciones más adecuadas en cada caso. ${ }^{8,11,15,32-34} \mathrm{El}$ hallazgo exclusivo de elevaciones de la TSH no debe conducirnos necesariamente a la asignación de un diagnóstico de por vida y mucho menos a la necesidad de un tratamiento indefinido, no siempre necesario o potencialmente perjudicial ${ }^{35}$. En ocasiones se pueden producir regresiones espontáneas a la normalidad, $\mathrm{o}$, incluso, puede ser válido mantener una "actitud expectante" o "vigilancia activa", clínica y analítica hasta disponer de criterios suficientes de beneficio esperable del tratamiento en cada paciente ${ }^{35}$.

En conclusión, pensamos que, en nuestro medio, la prevalencia de hipotiroidismo subclínico estimada a partir de la prescripción de hormona tiroidea es elevada, sobre todo en mujeres y personas de mayor edad, aunque se publican cifras variables en función del tipo de estudio. La forma autoinmune es la más habitual y, aunque parece más prevalente en el medio urbano, no podemos destacar diferencias evidentes en función de las variables geográficas estudiadas. El nivel de detección y porcentaje de pacientes bien controlados es adecuado y esto puede influir en la menor frecuencia de cuadros de hipotiroidismo clínico observados en la consulta. Por otra parte, la informatización de diversos

\footnotetext{
REV CLÍN MED FAM 2012; 5 (2): 89-96
} 
aspectos, analíticos, clínicos y farmacológicos, permite obtener datos con relativa facilidad, si bien no podemos prescindir de los estudios poblacionales, dado que éstos no están sometidos al sesgo de los institucionales.

\section{BIBLIOGRAFÍA}

1. Sender Palacios MJ, Vernet Vernet M, Franco Murillo A, OrtegaTallón MA, Pérez López S, Faro Colomés M. Estudio de patología funcional tiroidea en la población adulta de un centro de Atención Primaria. Medifam. 2002; 3:184-90.

2. Ibarz Excuer M, Sierra i Majem L, Gascó Eguiluz E, Serna Arnaiz MC, Vázquez Torguet A, Peremiquel Lluch M. Prevalencia de trastorno de la función tiroidea en la provincia de Lleida. Aten Primaria. 1999; 24:475-9.

3. Torroba Arlegui I, Puigdevall Gallego V, Laudo Pardos C, San Martín Barrio L. Disfunción tiroidea subclínica en atención primaria. Aten Primaria. 1998; 21:245-50.

4. Sender Palacios MJ, Vernet Vernet M, Pérez López S, Faro Colomés M, Rojas Blanc M, Pallisa Gabriel M. Enfermedad funcional tiroidea en la población de edad avanzada. Aten Primaria. 2004; 34:192-7.

5. Díez J, lbars MT. Prevalencia de disfunción tiroidea en ancianos. Med Clin (Barc). 2005; 124:437-9.

6. Nandalal B, Thomas R, Brown T, Ronald F Parish. Thyroid dysfunction in adults over age 55 years. A study in urban US community. Arch Intern Med. 1990; 150:785-7.

7. Bjoro T, Holmen J, Kruger O, Midthjell K, HunstadK K, Schreiner $T$, et al. Prevalence of thyroid disease, thyroid dysfunction and thyroid peroxidase antibodies in a large, unselected population. The Health Study of Nord-Trondelag (HUNT). Eur J Endocrinol. 2000; 143: 639-47.

8. Corrales Hernández JJ, Alonso Pedrol N, Cantón Blanco A, Galofré Ferrater JC, Pérez Pérez A, Lajo Morales T, et al . Guía clínica del diagnóstico y tratamiento de la disfunción tiroidea subclínica. Endocrinol Nutr. 2007; 54: 44-52.

9. Pérez Unanua MP, Mateo Pascual C, Muñoz González Y, Ruiz Begué M, Ortega Inclán N. Manejo de la patología tiroidea en Atención Primaria I. Cribado de patología tiroidea. Hipotiroidismo. SEMERGEN. 2008; 34: 450-4.

10. Villar HCCE, Saconato $H$, Valente $O$, Atallah AN. Thyroid hormone replacement for subclinical hypothyroidism. Cochrane Database of Systematic Reviews. 2007. Issue 3.Art No. CD003419. Pub 2. DOI 10.1002/14651858.

11. Samuels $\mathrm{MH}$. Subclinical Thyroid Disease in the Elderly. Thyroid. 1998; 8:803-13.

12. Bilous RW, Tunbridge WM. The epidemiology of hypothyroidism: an update. Baillières Clin Endocrinol Metab. 1988; 2: 531-40.

13. Rivolta G, Cerutti R, Colombo R, Miano G, Dionisio P, Grossi E. Prevalence of subclinical hypothyroidism in a population living in the Milan metropolitan area. J Endocrinol Invest. 1999; 22:693-7.

14. Cooper DS. Subclinical hypothyroidism (clinical practice). N Engl J Med. 2001; 354: 260-5.

15. Valentí G, Fabbo A. Subclinical hypothyroidism in the elderly. Arch Gerontol Geriat, suppl 5. (1996): 585-92.

16. Canaris GJ, Manowitz NR, Mayor G, Ridgway EC. The Colorado thyroid disease prevalence study. Arch Intern Med. 2000;160: 526-34.

17. Sawin CT, Castelli WP, Hershman JM, McNamara P, Bacharach P. The aging thyroid: thyroid deficiency in the Framingham Study. Arch Intern Med. 1985; 145: 1386-8.

18. Hunter I, Greene SA, MacDonal TM, Morris AD.Prevalence and aetiology of hypothyroidism in the young. Arch Dis Child. 2000; 83: 207-10.

19. De Santiago Corchado M. Hipotiroidismo. Medicine 2004; 9(14): 846-55.

20. Toni M, Anda E, Pineda J, Martínez de Esteban JP, Ollero MD. The effects of amiodarone on the thyroid. An Sist Sanit Navar. 2009; 32:363-70.

21. Morant Ginestar C, Criado-Álvarez JJ, García-Pina R, Pérez Garrido B. Estimación de la prevalencia del hipotiroidismo enEspaña a partir del consumo de hormonas tiroideas (1996-1999). Rev Esp de Salud Pública. 2001; 75: 337-44.

22. Díaz Madero A, López Ferreras A. Estimación de la prevalencia del hipotiroidismo en Castilla y León y su evolución desde 1992 al 2000 a través del consumo de hormonas tiroideas. Rev Esp Salud Pública. 2001; 75: 34552

23. Braceras Izaguirre L. Markuerkiaga Zubigarai M, Elizondo López de Landache I. Estimación de la prevalencia de hipotiroidismo en la Comunidad Autónoma del País Vasco a partir del consumo de hormonas tiroideas (2000-2006). FAP. 2007; 5: 49-54.

24. Serna Arnaiz MC, Galván Santiago L, Gascó Eguiluz E, Manrique M, Foix Oña MM, Martín Gracia E. Estimación de la prevalencia del hipotiroidismo en Lleida a partir de la prescripción de hormonas tiroideas. Rev Esp Salud Pública. 2003; 77: 405-10.

25. Sempere Verdú E, Feliu Sagala M, Hernandez Ruiz R, Ajenjo Navarro A. Prevalencia del hipotiroidismo tratado en la población adulta. Aten Primaria. 2005; 35: 163.

26. Ayala C, Cozar MV, Rodríguez JR, Silva H, Pereira JL, García-Luna PP. Enfermedad tiroidea subclínica en la población anciana sana institucionalizada. Med Clin (Barc). 2001;117:534-5.

27. Díez JJ, Molina, Ibars MT. Prevalence of thyroid dysfunction in adults over age 60 years from an urban community. Exp Clin Endocrinol Diabetes. 2003;111:480-5.

28. Maestro C. E, Albert C, V. Disfunción tiroidea en mayores de 75 años: Estudio en un colectivo rural no institucionalizado de Guadalajara. Rev Esp Geriatr Gerontol. 2005; 40: 14550.

29. Torné Coll M, Azagra-Ledesma R, Espina Castilla A, Vargas Vilardosa E, Reyes-Camps A. Hipotiroidismo subclínico en la consulta de Atención Primaria. Aten Primaria. 2006; 37: 175-76.

30. Peris Roig B, Atienza Herráis N, Merchante Alfaro AA, Calvo Rigual F, Tenías Burillo JM, Selfa Moreno S, et al. Bocio endémico y déficit de yodo: ¿sigue siendo una realidad en España? Ann Pediatr (Barc). 2006. 65: 234-40.

31. Díez JJ. Hypothyroidism in patients older than 55 years: an analysis of the etiology and assessment of the effectiveness of therapy. J Gerontol Med Sci. 2002; 57A :M315-20.

32. Lucas Martín AM. Hipotiroidismo subclínico: tratar o no tratar. Med Clin (Barc). 2004;122:182-3.

33. Klubo-Gwiezdzinska J, Wartofsky L. Thyrotropin blood levels, subclinical hypothyroidism, and the elderly patient. Arch Intern Med. 2009; 169(21): 1949-51.

34. M. Hornillos Calvo y J.G. Yela Gonzalo. Hipotiroidismo en el anciano. SEMERGEN. 2002; 28:137-44.

35. Rugge B, Balshem $H$, Sehgal R, Relevo R, Gorman P, Helfand M.. Screening and treatment of subclinical Hypothyroidism or Hyperthyroidism. Comparative effectiveness review $\mathrm{n}^{\circ} 24$ (Prepared by the Oregon Evidence-based Practice Center). AHRQ Publication $\mathrm{N}^{\circ}$ 11(12)-EHCO33-EF. Rockville, MD: Agency for Healthcare Research and Quality. Octuber 2011. Disponible en: www. effectivehealthcare.ahrq.gov/reports/final.cfm 